ÉGYPTE monde arabe

\section{Égypte/Monde arabe}

30-31 | 1997

Les visions de l'Occident dans le monde arabe

\title{
Ivresse et rapport à l'occidentalisation au Maghreb
}

Bars et débits de boissons à Tunis

\section{Emmanuel Buisson-Fenet}

\section{(2) OpenEdition}

Journals

Édition électronique

URL : https://journals.openedition.org/ema/1660

DOI : $10.4000 /$ ema. 1660

ISSN : 2090-7273

Éditeur

CEDEJ - Centre d'études et de documentation économiques juridiques et sociales

Édition imprimée

Date de publication : 30 septembre 1997

Pagination : 303-320

ISSN : 1110-5097

Référence électronique

Emmanuel Buisson-Fenet, «Ivresse et rapport à l'occidentalisation au Maghreb », Égypte/Monde arabe [En ligne], 30-31 | 1997, mis en ligne le 08 juillet 2008, consulté le 07 juillet 2022. URL : http:// journals.openedition.org/ema/1660; DOI : https://doi.org/10.4000/ema.1660

Ce document a été généré automatiquement le 7 juillet 2022

Tous droits réservés 


\title{
Ivresse et rapport à l'occidentalisation au Maghreb
}

\author{
Bars et débits de boissons à Tunis
}

Emmanuel Buisson-Fenet

1 Tunis compte aujourd'hui quelques dizaines de lieux où de l'alcool est vendu et consommé : bars (en petit nombre), marchands de vin, restaurants liés ou non au tourisme, hôtels... Autant dire que l'on ne se trouve ni dans quelque capitale occidentale, où l'alcool fait partie de l'ordinaire de la vie sociale, ni dans l'un des - rares - pays musulmans où le commerce d'alcool fait l'objet d'une interdiction complète. Cette position médiane, qui ne relève pas de l'interprétation sociologique mais de l'observation la plus élémentaire, est très souvent occultée dans les discours relevés à Tunis $^{1}$, au sujet de la fréquence de la consommation d'alcool. Les acteurs pressentent immédiatement que la question de la fréquence de la consommation d'alcool implique la possibilité d'un jugement sur les contradictions entre le caractère illicite de cette pratique (du point de vue de la morale musulmane), et son degré de tolérance (dans les pratiques quotidiennes)*.

2 Avant même d'être posée en terme de "rapport à l'Occident », la fréquence de la consommation d'alcool soulève donc un problème de représentation de soi dans un pays musulman comme la Tunisie. C'est ainsi qu'il faut comprendre les deux stratégies rencontrées si fréquemment dans les entretiens pour caractérise les buveurs et leur statut : " personne ne boit, sauf des marginaux, des gens occidentalisés », réajustent la norme religieuse et la tolérance quotidienne dans un sens restrictif, en faisant de cette question un problème exogène. Les Tunisiens ne boivent pas, sauf quand ils ne sont plus vraiment Tunisiens. Bien entendu, on ne fait pas référence ici aux textes religieux mais à la règle admise par tous qui veut qu'un musulman ne doit pas consommer d'alcool. À l'inverse, il n'est pas rare d'entendre certains - notamment des buveurs affirmer que «tout le monde boit mais en cachette, ça ne se dit pas", recourant ainsi à un argument de l'hypocrisie sociale, très fréquemment utilisé au Maghreb dans les situations où les représentations de soi et les pratiques visibles ne semblent pas se correspondre. 
3 D'un point de vue factuel, la vérité est quelque part entre ces deux stéréotypes, et les Tunisiens (dans la capitale en tout cas) consommant de l'alcool assez régulièrement sont à la fois une minorité de la population masculine et une part non négligeable de cette dernière, ce que montre sans conteste une estimation du nombre de débits de boissons dans Tunis et sa banlieue. Il serait de peu d'intérêt de rechercher avec précision cette proportion si l'on souhaite réfléchir sur les pratiques des buveurs, tout comme la recherche du "véritable» chiffre de la délinquance - «le chiffre noir» (Herpin, 1973), par opposition à la délinquance relevée par la police -, n'a pas apporté en son temps de résultats probants à la sociologie américaine de la déviance.

4 Les deux stéréotypes relevés dans les discours des Tunisiens sur les buveurs sont révélateurs en revanche du décalage qui peut apparaître entre des pratiques quotidiennes et les représentations qui en sont fournies, particulièrement les représentations en public. Boire de l'alcool est possible, mais on ne saurait le faire devant tous, hors des lieux partagés par les buveurs; et surtout on ne saurait le revendiquer - acte réservé aux poètes et aux fous ${ }^{2}$... Il apparaîtra que ce décalage joue un rôle majeur dans la mise en scène de «l'Occident » à travers l'alcool, car elle rend possible une représentation "exogène » à la société tunisienne de la pratique des buveurs.

5 Les connexions entre la consommation d'alcool et la représentation de l'Occident sont cependant très variées : elles sont repérables à trois niveaux, pour trois ordres de problèmes pas nécessairement liés entre eux.

6 Historiquement, les débits de boissons se sont répandus à Tunis avec la colonisation. Comme dans la plupart des cités au Maghreb, ils se trouvent généralement dans la partie européenne de la ville, ou dans les villes de banlieue qui ont grandi à l'époque coloniale le long de la côte. Cela ne signifie pas que la consommation ou la production d'alcool était absente du Maghreb avant la colonisation, mais que les lieux publics qui lui sont associés sont liés à l'histoire de la présence occidentale. Les bars et les marchands de vin se rassemblent donc pour l'essentiel dans les anciens quartiers populaires et les lieux de passage de la ville européenne : entre la gare et l'artère principale de Tunis (l'avenue Bourguiba), ou encore à la Goulette, lieu toujours populaire de villégiature et ancienne ville de l'immigration italienne ${ }^{3}$.

7 Dans les discours sur l'alcool, il est très fréquent que la présence de ce dernier soit associée à une "occidentalisation des modes de vie", et par voie de conséquence, la pratique des buveurs peut être perçue comme exogène, «pas vraiment tunisienne » ou réservée à de rares personnes occidentalisées. Cette constitution de l'alcool comme un produit étranger entrânant une pratique exogène à la société tunisienne explique largement, comme on le verra, l'attitude et le comportement des buveurs, qui le savent pertinemment. Certains dispositifs peuvent contribuer à valider cette représentation : l'alcool est en vente dans certains supermarchés, fortement associés à un mode de consommation occidental, ou encore dans les lieux touristiques. Il n'en reste pas moins que ce discours ne correspond pas à des différences réelles, substantielles ${ }^{4}$ entre buveurs et non-buveurs: les premiers ne sont pas plus "occidentalisés » que les seconds ; cet argument sert avant tout à la disqualification de ceux qui consomment de l'alcool.

8 Quand on se rend dans un débit de boissons vendant ou non de l'alcool, les buveurs comme les non-buveurs (qui peuvent être une seule et même personne prise dans des circonstances différentes) choisissent ce lieu avec le plus grand soin, en fonction de son 
caractère plus ou moins « local » ou " occidental », ce qui implique une décoration, une clientèle et des comportements admis ou impossibles, particuliers à chaque type de lieu. Il existe donc aussi un "usage local et non-polémique" de la référence à l'Occident, suivant l'expression de Jean-Noël Ferriés

9 Chacun de ces aspects du "rapport à l'Occident", à travers l'une de ses productions qu'est l'alcool, peut servir à accréditer l'idée que l'acte de boire est un signe d'occidentalisation et de perte d'identité, ou encore que les buveurs sont atypiques ou marginaux. Cette représentation est d'autant plus répandue que la reconnaissance publique de la consommation d'alcool est exceptionnelle, et sa visibilité faible y compris dans les productions artistiques ${ }^{6}$. On voudrait montrer ici, à travers l'observation des pratiques en cours dans les lieux de consommation d'alcool, que l'univers social des buveurs est aussi intégré qu'il est possible à la société tunisienne environnante, à ses références culturelles comme à ses mécanismes de fonctionnement.

\section{Le choix du lieu : cafés, bars et restaurants}

10 Dans l'ensemble des pays du Maghreb, deux éléments structurent l'accès aux débits de boissons : d'une part, la présence ou non de boissons alcoolisées, et, de l'autre, la mixité ou l'existence d'une clientèle exclusivement masculine. Chacun sait ou apprend à reconnaître les différents débits de boissons en fonction de ces deux critères, et chacun sait parfaitement reconnaître et adapter aux circonstances le choix du lieu où se rendre. Les bars, par exemple, se distinguent largement des cafés, qui ne vendent pas d'alcool: ils sont pour la plupart situés au cœur de la cité, mais rien n'indique de l'extérieur que l'on est face à un bar. Il existe fort peu d'exceptions qui, à l'instar du " café de Paris ", au beau milieu de l'avenue Bourguiba, montre au public par ses larges baies vitrées des buveurs attablés. Pas d'enseigne au nom d'une célèbre marque de bière, et pour beaucoup, pas d'indice extérieur. Le vocabulaire est à l'image de cette discrétion des façades: le chrab, littéralement la boisson, désigne en fait le vin. Pourtant, les hommes savent pertinemment à quel type de débit de boissons ils ont affaire dans un lieu particulier, quelle clientèle est autorisée à y accéder, et dans quel créneau horaire. De même la question «tuchrub? "(tu bois ?), fait entrer, par sa forme euphémisée, dans ce jonglage permanent entre les lieux et les horaires. À l'inverse, les cafés peuvent être largement ouverts aux regards, et les longues terrasses sont fréquentes dans tous les quartiers de la ville.

11 Second critère de choix, les débits de boissons - cette expression étant entendue au sens le plus large - offrent sous certaines conditions la possibilité d'un accès aux femmes. Généralement, ces lieux sont perçus comme masculins, mais là aussi, chacun sait que dans certains lieux, l'entrée d'une femme ne posera pas de problème. C'est le cas dans les petits restaurants, les "fast-foods» qui se sont multipliés dans les quartiers aisés, mais c'est aussi le cas des cafés fréquentés par les touristes, ou bien des bars d'hôtels internationaux dans le centre de Tunis. L'identification supposée entre "lieux publics» au Maghreb et «lieux masculins », fréquente chez certains auteurs ${ }^{7}$, confond en fait l'énoncé prescriptif, que l'on peut toujours opposer à une femme pour lui signifier qu'elle n'est pas admise dans une circonstance bien précise, et les pratiques d'acteurs reconstruisant à chaque moment la frontière entre « lieux mixtes » et « lieux masculins». Selon l'expression de Goffman (1973, p. 105), il s'agit en fait d'un « comportement régional ", ajusté et limité à des circonstances précises. Par exemple, 
un bar de la rue Garibaldi est exclusivement réservé aux hommes pendant les heures où la vente d'alcool est autorisée, et constitue un des lieux de rencontre favoris des jeunes couples aux autres heures de la journée.

12 Combinés, ces deux critères de sélection offrent donc quatre possibilités pour classer les débits de boissons, chaque groupe possédant des caractéristiques assez semblables. La présence de femmes et la possibilité de consommer de l'alcool discriminent aussi des lieux qui n'usent pas de références à l'Occident au même degré. Il ne faut y voir aucun hasard: ces deux critères servent dans bien d'autres occasions à caractériser précisément l'Occident, ou les signes d'occidentalisation à l'intérieur de la société (Zannad, 1984 ; Petonnet, 1972).

13 Commençons par les lieux qui excluent la présence de femmes comme la vente de boissons alcoolisées. Il s'agit essentiellement des cafés de quartier, parfois appelés cafés maures suivant une ancienne opposition avec les cafés "à l'européenne ", même si la distinction n'est plus vraiment opératoire aujourd'hui, dans la mesure où le mobilier européen s'est largement répandu. On y sert café, thé et boissons fraîches, il est possible d'y fumer la shlha (pipe à eau), d'y jouer aux cartes, etc. Ces cafés dominent la médina, et quadrillent les quartiers populaires : lieux ouverts à tous les hommes de la houma (du quartier), où l'on se retrouve presque chaque jour. Lieux de forte interconnaissance, la présence de femmes y serait donc source d'ennuis ou de gêne, et les jeunes filles évitent scrupuleusement de s'y montrer.

14 Il en va tout autrement de nombre de débits de boissons, qui, pour des raisons très diverses, présentent une réelle mixité, tout en n'offrant pas de boissons alcoolisées. Ainsi, dans les "pâtisseries» et chez nombre de vendeurs de casse-croûte et de restauration rapide, la présence des femmes se justifie par la possibilité d'y manger (même si l'on peut se contenter d'y boire quelque chose) ; les plus récents de ces lieux, particulièrement dans les quartiers aisés, proposent une décoration et un aménagement intérieur rappelant les «fast-foods ». La décoration occidentale est alors un argument de vente. Au contraire, certains cafés en nombre assez limité usent délibérément d'une décoration «traditionnelle ", les chaises étant remplacées par des nattes, les tables par des meïda (table basse). Ces cafés se trouvent dans des lieux touristiques ayant préservé l'habitat urbain traditionnel : la médina de Tunis, ou Sidi Bou Saïd sur la côte. À l'inverse de l'exemple précédent, la reconstruction d'une "décoration locale ", pour les besoins du tourisme, a permis aux jeunes, en couple ou en groupes mixtes, de les réinvestir largement en dehors de la saison touristique. Ces cafés ont perdu en effet toute fonction de "sociabilité de quartier ", et donc le contrôle social afférent; on y vient de loin pour l'agrément du cadre et parce que l'on sait bien que ces lieux acceptent une clientèle mixte. En pratique, la « couleur locale » joue donc exactement le même rôle que le « décor international » des fast-foods, elle permet de sortir des contraintes attachées au café de quartier.

Les bars des hôtels touristiques et, dans une moindre mesure, les restaurants de bonne tenue (fréquentés également par les touristes) présentent des caractéristiques assez similaires : il est possible d'y boire de l'alcool et la présence des femmes est acceptée. On retrouve une justification assez semblable à l'exemple précédent, la fonction touristique de ces lieux ayant en quelque sorte "extra-territorialisé » les comportements admissibles. La présence d'occidentaux, même si elle est conditionnelle, et le fonctionnement suivant des normes internationales rend possible des comportements qui ne seraient pas tolérés ailleurs. Durant toute la belle saison, les 
restaurants de la ville côtière de La Goulette reçoivent par exemple à la fois des familles et des groupes de buveurs, en terrasse et aux yeux de tous. Mais ces lieux ne constituent pas un monde séparé et homogène, et les limites ou les seuils à ne pas dépasser sont parfois difficiles à tracer, dépendant parfois largement de la bonne volonté du serveur. Le milieu social, la tenue des clients jouent un grand rôle dans ces négociations, dans la mesure où ils engagent la "réputation» du lieu; les prix pratiqués étant assez largement au-dessus des prix habituels des cafés et des bars, la sélection de la clientèle se fait d'elle-même.

Les bars et les bars-restaurants constituent un groupe assez différent, dans la mesure où ils sont fréquentés presque uniquement par des Tunisiens, et la présence de femmes, $\mathrm{y}$ est exceptionnelle. Les bars proprement dits où l'on sert uniquement de l'alcool aux heures autorisées sont peu nombreux, et situés pour l'essentiel dans le centre de Tunis, dans les rues adjacentes à l'avenue Bourguiba, et aussi dans les villes côtières de la banlieue tunisoise. Certains restaurants, appelés souvent bar-restaurant, fonctionnent en fait comme des débits de boissons : on y prend un plat pour deux, ce qui constitue un prétexte à la présence dans le "restaurant " et un moyen simple d'outrepasser les horaires légalement réservés aux bars. On y consomme en fait de la bière ou du vin, et ces lieux dessinent la même carte que les bars. Nous reviendrons longuement sur les comportements visibles dans les bars, mais retenons pour l'instant ce qui fait la spécificité de ces lieux. Symétriquement à l'exemple précédent, le fait qu'ils ne soient pratiquement pas fréquentés par des non-Tunisiens renvoient à deux de leurs propriétés, l'absence des femmes et la discrétion de ces lieux. La plupart des bars ou des bars-restaurants sont en effet méconnaissables de la rue, sans vitrine en façade ni enseigne reconnaissable. Il faut bien les connaître pour s'apercevoir de leur présence. S'ils gardent parfois des signes de l'héritage colonial, leur nom pour l'essentiel ${ }^{8}$, on est donc bien en face de lieux fonctionnant dans une logique profondément locale, sans référence particulière à des équivalents occidentaux.

17 À Tunis, et plus généralement au Maghreb, la classification des débits de boissons doit inciter à la prudence envers tout énoncé qui identifierait lieux publics et lieux masculins, ou encore consommation d'alcool et occidentalisation des lieux ou des comportements. Toutes les combinaisons sont possibles entre ces différents critères, et il n'y a rien de surprenant dans cette logique à voir par exemple une femme boire une bière avec des amis dans un lieu public, alors qu'elle ne pourrait pas même prendre un café dans un autre. L'adaptation complexe à des horaires sévèrement réglementés ${ }^{9}$, aux variations entre des lieux en apparence très proches, signale que les frontières sont généralement un enjeu et un objet de négociation. Il est toujours possible d'essayer de les outrepasser, mais on s'expose alors à des sanctions verbales: refus de service, admonestation voire éviction pure et simple. Les individus anticipent constamment ces réactions jusqu'à ajuster leurs comportements à ce qui est admis dans des circonstances données. En d'autres termes, la consommation d'alcool n'est pas sanctionnée quand elle respecte des "cadres prédéfinis»; ce qui est lourdement sanctionné en revanche, c'est la faute de goût et l'ignorance délibérée ou involontaire de ces conventions. Dans ce cas seulement, on entendra des arguments généraux ( les femmes ne doivent pas boire", "nous ne servons pas d'alcool car c'est illicite»), arguments qui n'ont aucune incidence le reste du temps. Dans ce système fondé sur la discrétion et l'ajustement implicite des comportements, la dénonciation de la consommation d'alcool comme acte "non tunisien ", "non musulman » ou encore, signe d'une occidentalisation regrettable, trouve toute sa puissance, alors même que 
l'observation la plus élémentaire de la vie sociale dans les débits de boissons montre, au contraire, la réelle intégration de ces lieux dans la société tunisienne.

\section{Les bars : un lieu de construction de l'identité}

Des différents types de débits de boissons repérés à Tunis, les bars - et les barsrestaurants - forment le groupe le plus intéressant pour réfuter l'idée d'une "occidentalisation des comportements", via des produits de consommation comme l'alcool. Ces lieux sont en effet, comme on vient de le voir, fréquentés uniquement par des Tunisiens, et ne sont pas liés au développement du tourisme. Si les bars restent très discrets de l'extérieur, il ne faut pas en déduire que la consommation d'alcool est un acte honteux, fait en quelque sorte "en dehors» des autres pratiques sociales. Au contraire, la description des comportements masculins dans les bars met à jour une situation paradoxale : les buveurs sont très discrets sur leurs pratiques, en particulier envers leur famille, mais celles-ci présentent de nombreuses homologies avec les comportements les plus attendus, et les plus valorisés chez un homme. Tout se passe donc comme si les bars étaient l'un des hauts lieux de l'affirmation de l'identité masculine, alors précisément que ce lieu se doit de rester invisible par rapport au monde social environnant.

Que se passe-t-il donc lorsque l'on décide de franchir la porte d'un bar ? D'abord, on n'y entre pas seul, car boire est un acte collectif, un plaisir qui se partage. D'ailleurs, les buveurs isolés - uniquement des hommes, la majorité ayant entre 30 et 40 ans cherchent souvent à lier conversation avec leurs voisins, à interrompre la mélancolie qui semble toucher l'acte solitaire. Car la bière coûte cher et les plus jeunes, les plus désargentés, préfèrent l'acheter en magasin et la boire sur la plage la nuit, ou dans tout autre endroit à l'abri des regards. Quant aux hommes plus âgés, ils ont souvent quitté ce monde social depuis peu : combien de fois ai-je entendu la même histoire familiale racontée par un jeune homme, "mon père aussi était un grand buveur, comme moi, mais maintenant il en a fini avec la boisson, il ne sort plus ». Le bar ne concerne qu'une petite cohorte masculine, mais surtout un âge de la vie assez bien délimité, de la fin de l'adolescence à la maturité. On y entre parfois par bravade, pour montrer qu'on est un homme, et l'on en sort quand les enfants viennent à prendre la place et que l'on n'a, justement, plus rien à prouver.

20 La quasi-totalité des buveurs consomment de la bière locale dans les bars, et parfois du vin tunisien dans les bars-restaurants. Les produits importés d'Occident sont exceptionnels dans ces circonstances collectives : ils sont très chers et plutôt réservés aux brasseries des hôtels internationaux, ou à l'usage individuel d'une clientèle aisée. L'opposition entre la consommation collective de la bière, au bar, et la consommation individuelle de boissons importées (comme le whisky) recoupe une opposition sociale entre la clientèle populaire - qui exclut de boire chez soi et souvent de boire seul - et un mode de consommation bourgeois, où boire est un acte privé.

21 Une fois installés à une table, la première règle des buveurs est de rester ensemble au bar aussi longtemps que possible. Il est exceptionnel de rentrer ici pour «boire une bière ", ou même deux, en particulier chez les hommes encore célibataires : on occupe les lieux jusqu'à l'ivresse, si les finances le permettent. Le bar n'a pas cette fonction de sociabilité quotidienne que connaît le café : y entrer, c'est généralement accepter de ne pas savoir quand l'on en sortira, ni même comment. Emporté avec un groupe d'amis 
dans un bar, il n'existe pas vraiment de "procédure d'arrêt " à la consommation d'alcool: on boit tant qu'il reste de l'argent pour cela, chez les jeunes tout particulièrement. La recherche de l'ivresse commune participe de cet état d'esprit, mais elle s'explique surtout par la nécessité pour chacun de montrer sa prodigalité, la générosité de «l'homme véritable » qui sait ne pas compter. Générosité, simultanément envers ses amis - il serait déshonorant de laisser partir quelqu'un de la table sous le prétexte qu'il n'a plus d'argent - et générosité de caractère, de «bon vivant » dirait-on, qui appréciera toujours une nouvelle tournée. La valorisation de ces sentiments dépasse largement l'univers du bar: on retrouve le même type d'attitude, la même importance accordée à la générosité quand on se présente dans un cadre plus familial.

Lorsque des compagnons de bar ont un peu plus d'argent, la soirée sera poursuivie après la fermeture des débits de boissons dans un bar-restaurant, jusqu'à ce que plus personne ne puisse offrir une suite. Raison peut-être de la crainte étatique du bar, manifestée par les politiques répressives envers les débits de boissons : les hommes y pénètrent souvent pour ne plus avoir à compter, pour montrer à chacun ce dont ils sont capables. Régulièrement, le cinéma maghrébin nous offre une "scène de genre ", évoquant la recherche de cette ivresse collective et sans limite, surtout chez les jeunes hommes ${ }^{10}$. La dérision enfin est toujours présente, pendant picaresque à la fraternisation entre les consommateurs d'une même table. Elle est peut-être la raison secrète de la recherche de l'ivresse, par la possibilité qu'elle engendre de se laisser totalement emporter, gagner par ces moqueries perpétuelles, sans plus avoir à adopter une attitude défensive et à " prendre au mot » les propos des autres, à réagir à toute insulte qui mettrait en cause son honneur. L'ivresse déconnecte, pour un temps, le monde du bar de son environnement social, et offre aux hommes l'occasion de se moquer d'eux-mêmes.

Ces quelques observations doivent suffire à montrer que, si la fréquentation d'un bar ne peut faire l'objet d'une revendication, cela ne signifie pas un instant que les buveurs aient un comportement marginal au sein de la société tunisienne, ni même que leur attitude soit toujours considérée comme illégitime par les non-buveurs. À l'extérieur du bar, la discrétion est indispensable, mais elle ne signifie pas que la pratique ellemême soit condamnée : si un père et un fils, et plus largement deux membres d'une même famille, peuvent savoir tous deux qu'ils fréquentent les bars, ils éviteront soigneusement d'aller dans les mêmes lieux. Les bars ne constituent donc pas précisément des « lieux publics ", au sens où on l'entend en Occident, car leur accès est limité et que l'on s'y retrouve en groupe, entre amis. Il faudrait plutôt parler de « lieux réservés ", suivant l'expression de M. Bozon et F. Héran (1987-1988), autour d'un public particulier. On comprend mieux pourquoi peuvent se montrer, dans le bar, des comportements d'ivresse qui n'auraient pas leur place dans des lieux publics, mais aussi dans le cadre privé de la famille.

Regroupant les individus dans une chaîne d'interconnaissance, le bar rend visible l'existence au Maghreb de différentes modalités de l'affirmation de soi pour les hommes, de sa « virilité » (rujûliyya).L'une est réservée aux relations avec des inconnus, et par exemple au comportement dans des lieux publics comme la rue : c'est le modèle souvent développé de la virilité agonistique ${ }^{11}$ basée sur le " sens de l'honneur », comme la désigne $P$. Bourdieu. Rares sont les cas où un tel type de comportement apparaît dans les bars, sinon dans des disputes, des bagarres qui demeurent l'exception à Tunis. Il en irait tout autrement dans la rue ou dans des lieux ouverts à tous. 
Symétriquement, il existe aussi une modalité généreuse et pacifique de la virilité, vertu recherchée chez ses amis, certes partagée avec les compagnons de bar, mais également en bien d'autres circonstances ${ }^{12}$. La générosité et le désintéressement envers les autres, le refus de calculer sont de cet ordre, et l'ivresse y participe largement. Aller boire, c'est aussi rechercher cette forme de fraternisation masculine, se retrouver «entre soi ", hors des contraintes du monde social environnant, hors de tout calcul. D'autres exemples, tirés de l'anthropologie méditerranéenne, vont dans le même sens : ainsi la notion grecque de kefi, à la fois ivresse et qualité de l'homme généreux, caractérisant "celui qui a du cœur» tout autant que celui qui sait vivre ${ }^{13}$. À la différence de la première conception, elle est donc réservée aux relations d'interconnaissance, que l'on trouve par exemple dans les bars.

Il n'est pas nécessaire de développer cette opposition ici, signalons simplement qu'elle permet $d$ 'insister sur la forte intégration des règles d'interaction masculines visibles dans les bars à ce qui est valorisé et attendu d'un homme en diverses circonstances. Le paradoxe, c'est donc que l'acte de boire est socialement peu accepté en dehors de lieux strictement délimités comme les bars, alors que les interactions qui se nouent autour de l'ivresse commune sont fortement valorisées, et peuvent être montrées et mises en scène si le lien avec la dive bouteille est masqué.

\section{L'ivresse et ses masques : la valorisation d'une émotion non revendicable}

Suivant le niveau d'observation choisi, la consommation d'alcool et les émotions qui lui sont attachées comme l'ivresse vont donner lieu à des constructions très différentes, et même contradictoires. De ce fait, la référence à l'Occident ne prend pas toujours la même place: inévitable quand il s'agit d'élaborer une représentation publique de l'alcool, elle est très secondaire pour les individus qui fréquentent les bars.

Dans des circonstances publiques, la consommation d'alcool n'est pratiquement jamais reconnue par les buveurs, ni même montrée (à l'exception du cinéma). Les articles de la presse tunisienne sur ce sujet - y compris sous un angle prophylactique - sont exceptionnels. Il n'est pas étonnant alors, comme le justifie partiellement l'histoire de la colonisation, que la consommation d'alcool soit attribuée à un modèle exogène, signe d'une occidentalisation regrettable des comportements ${ }^{14}$. Le roman La Fêlure de Lhabib Chebbi (1985), en délivre une version dramatisée : le narrateur est un cheikh reconnu dans la médina de Tunis, à la fin du siècle dernier. Profondément tourmenté par l'épidémie de choléra qui sévit alors dans la ville, il se rapproche peu à peu de la ville européenne, en plein essor, et finit par fréquenter assidûment ses bars à mesure que ses convictions religieuses se dégradent. Devenir buveur est alors, simultanément pour le narrateur, un signe de désocialisation et de perte de son identité tunisienne. Symétriquement, c'est l'occidentalisation de la ville qui a généré ce comportement.

Il ne faudrait cependant pas se tromper sur la portée de cette condamnation publique de la consommation d'alcool. On l'a déjà souligné précédemment, il s'agit moins de rejeter les buveurs, que de leur demander la discrétion pour respecter les convictions d'autrui, surtout dans le contexte familial. Le fait de boire n'entraîne donc pas de véritable culpabilité, surtout quand il s'agit, chez les jeunes, d'un moyen de se construire. C'est pourquoi au bar, mais souvent aussi au café, au hammam et dans les 
lieux réservés aux hommes, les personnes d'une même famille évitent souvent de se rencontrer. Un père et son fils, ou même deux frères ou deux cousins, veillent scrupuleusement à fréquenter des lieux séparés. Rencontrer dans un bar un membre de sa famille, quelqu'un avec qui l'on est lié autrement que par la libre amitié, entraîne gêne et déplaisir, le sentiment que l'on ne pourra plus exactement être soi-même en sa présence. Situation très fréquente chez les jeunes hommes qui, tout comme leur père, peuvent par exemple fumer et même boire de l'alcool, mais ne le feront jamais devant lui. « C'est par respect » dit-on alors, car la conduite juste ne repose pas ici sur le fait de ne pas commettre des actes illicites - père et fils n'évoqueront pas le sujet ensemble mais sur la pudeur nécessaire, la discrétion envers les proches. Morale de la visibilité et non de la culpabilité, qui laisse en fait beaucoup de marge de manœuvre aux individus ; mais elle est aussi à l'origine de bien des cas d'incompréhension entre Occidentaux et Maghrébins, par la séparation qu'elle induit entre les convictions individuelles ( $«$ je ne vois aucun mal à boire », par exemple), et le respect des conventions («j'accepte de m'en cacher »).

Les buveurs acceptent généralement d'avoir à masquer leur comportement, et on a parfois l'impression que cet aspect fait presque partie du plaisir pris dans la fréquentation des bars. Il y a une part de vérité dans ce constat, car le bar offre une réelle occasion de s'autonomiser du contrôle social exercé par l'environnement familial. Fréquenter le bar permet d'échapper au regard des autres, choisir véritablement, dans une vie souvent souterraine au regard de sa vie publique et familiale, quelles relations l'on souhaite engager et qui l'on veut être.

31 En un mot, le passage discret, inénarrable dans un bar, est l'un de ces moments où chacun se sent maître de ses actes. Jean-Noël Ferrié (1991) notait avec beaucoup d'àpropos, dans un article consacré à la construction de l'individu au Maghreb, que l'identité individuelle ne s'y trouvait ni absente ni même " amputée » comme l'on peut trop fréquemment l'entendre. Simplement, les procédures par lesquelles chacun expérimente son autonomie individuelle ne prennent pas les mêmes chemins qu'en Occident : il y a là une manière propre de se construire, selon sa formule, une autre " évidence de l'individu ». La conception occidentale de l'individu repose sur le respect de la sphère privée, espace d'autonomie pour chacun. Au contraire, il est beaucoup plus fréquent au Maghreb de se construire en trouvant le moyen d'échapper à la sphère privée, et d'abord familiale, pour s'autonomiser dans des lieux publics ou semi-publics dont le bar offre un exemple. La seule condition est de faire preuve de la plus grande discrétion : plus la séparation est grande entre le monde des amis et le monde familial, plus on garantit sa propre liberté. Il existe pour sûr bien d'autres moyens de s'autonomiser, la référence à l'Occident et le choix ou la recherche d'une migration vers cet ailleurs étant l'une de ces possibilités, mais le bar présente l'avantage de rester disponible et d'être, par ses caractéristiques mêmes, très peu visible du reste de la société.

Voilà pourquoi l'on ne peut, au bar, fréquenter des membres de son lignage, voilà aussi l'une des raisons pour lesquelles, bien au-delà du petit monde des débits de boissons, l'apprentissage de sa vie d'homme passe si souvent par la possibilité de s'échapper de la vie familiale. Les jeunes Tunisiens, dans le monde urbain en tous cas, quittent maintenant très tardivement le domicile paternel, du fait du recul de l'âge au mariage autant que des coûts prohibitifs des loyers; mais très tôt, ils apprennent à y passer le moins de temps possible, pour rejoindre leur groupe d'amis - les cliquât, les «cliques » 
qui ont leurs habitudes dans chaque quartier. L'essentiel de la journée, et même de la nuit, est passé avec des amis, en échappant au contrôle familial. Certains de mes amis, parfois trentenaires, menaient ainsi une existence semi-nomade, dormant rarement chez leurs parents, y passant dans la journée de façon très aléatoire le temps d'un repas. Ce comportement est généralement approuvé, et même valorisé, à l'égard des garçons - on s'inquiète plutôt d'un homme qui passerait son temps à la maison, attitude réservée aux hommes âgés. La vraie vie est ailleurs, parfois dans les bars et presque toujours dans les cafés, parmi ses pairs et hors du contrôle lignager.

Aucun discours ne vient appuyer ce rôle des bars dans la construction de l'identité individuelle, et contrebalancer la condamnation publique de la consommation d'alcool, si bien qu'il peut largement passer inaperçu. Pourtant, c'est l'un des plus solides moteurs de l'attrait que ce lieu exerce, et c'est aussi la première raison de sa pérennité, bien au-delà du sort réservé aux survivances de la colonisation. La discrétion qui entoure les bars fait donc, en quelque sorte, partie de leur fonction.

Si l'ivresse associée à l'alcool ne doit pas être manifestée à l'extérieur des bars, sans risquer des sanctions sociales ou même légales ${ }^{15}$, le sentiment de l'ivresse peut en revanche être mis en scène et fortement valorisé quand il apparaît dissocié de l'alcool. La musique (ou la danse) est souvent, au Maghreb, un moyen d'exprimer son enivrement, sous la forme d'une expérience collective où la générosité envers autrui joue également un grand rôle. Tout comme la bière délivre les buveurs de la nécessité du contrôle de soi, la musique peut également être libératrice. Dans les soirées musicales par exemple, les 'awadât autour du luth et de la darbouka, la décision de danser est une expérience assez similaire aux démonstrations de générosité des buveurs: assis jusqu'à présent pour écouter la musique, quelqu'un se lève soudainement, comme emporté par la mélopée. Les musiciens le savent bien, tant leur art implique un sens de la tension, de l'attente soigneusement entretenue dans l'introduction des chansons, jusqu'au moment où le rythme entraînera vers la danse. Cet emportement, cette ivresse qui semble "prendre " le nouveau danseur est très valorisée, et provoque souvent les applaudissements et les encouragements du public.

La notion de tarab exprime bien la liaison qui existe entre la musique, l'ivresse et l'ouverture aux autres : elle désigne à la fois la gaieté, la joie et le fait de «s'extasier devant la musique, la poésie $»^{16}$. Le terme s'applique d'ailleurs aussi bien au sentiment collectif de l'auditoire qu'à l'émotion exprimée par le chanteur, lorsque la mélopée " monte », et que lui font écho à ce moment précis les clameurs soudaines du public. Si elle ne fait pas partie du vocabulaire propre aux débits de boissons, elle donne une bonne idée du type d'émotion valorisé par les buveurs, tant la recherche de cette forme d'emportement hors de soi est proche dans les deux circonstances. La valorisation de l'expérience de l'ivresse, dans ses formes culturelles les plus légitimes (par la poésie ou la musique), n'est donc pas étrangère au type d'expérience recherchée par les buveurs, dans un tout autre registre.

Les distinctions à l'oeuvre entre différents genres musicaux donnent une assez bonne idée de la frontière, poreuse et pourtant bien réelle, entre l'ivresse la plus légitime et la plus valorisée, et ses formes déniées. Les deux répertoires de la musique algérienne les plus connus, le raï oranais et le cha'bi algérois, expriment très souvent des émotions liées à l'ivresse, mais dans des registres bien distincts ${ }^{17}$. Le raï chante de façon très directe l'alcool, les relations avec les femmes, ou encore la possibilité d'un départ vers l'Occident (thème récurrent du visa). Il s'écoute rarement en famille, car il met en 
scène des modalités illégitimes de l'ivresse, et est parfois perçu comme un genre musical occidentalisé, par ses thématiques comme par son instrumentation «moderne». Au contraire, le cha'bi peut être écouté en toutes circonstances, et euphémisme systématiquement les références à l'ivresse (ivresse de la pureté des sentiments amoureux, par exemple). Ce travail de sublimation des circonstances liées à l'ivresse n'exclut pas les doubles sens ou les sous-entendus, et les encouragerait même chez l'auditeur. Ces deux genres musicaux chantent donc tous deux l'ivresse, sous deux modalités distinctes: ils rappellent que cette émotion peut être très valorisée, à condition que les moyens d'y parvenir restent dans le vague. Il n'est pas rare de voir, dans les mariages, les chanteurs de cha'bi boire discrètement de l'alcool entre deux chansons, et à vrai dire, nul ne leur en tiendra rigueur s'ils ne se font pas remarquer...

La consommation d'alcool manifeste bien là ses ambiguïtés : le produit reste illégitime au Maghreb, mais les émotions ou les attitudes qui lui sont associées participent pleinement du monde social environnant. Il n'est donc pas surprenant de voir si souvent surgir, surtout de la part des jeunes, la dénonciation d'une « hypocrisie » qui serait propre à ces sociétés. L'argumentation repose sur l'opposition entre un Occident où les choix individuels des modes de vie, des rapports sentimentaux peuvent être rendus publics, et des sociétés arabes hétéronomes, où de nombreuses pratiques seraient tolérées ou répandues, à condition de rester secrètes. La dénonciation de l'hypocrisie concerne autant la consommation d'alcool, que l'homosexualité ou les rapports amoureux avant le mariage, voire l'usage du tabac. Cet argumentaire n'est pas anecdotique, bien qu'il n'ait guère intéressé la sociologie jusqu'à présent. Il construit négativement d'abord une image des sociétés maghrébines par rapport à l'Occident, de l'intérieur même de ces sociétés. Ce discours est donc générateur de souffrance sociale, à une époque où les possibilités de migration sont restreintes et alors même que la visibilité de l'Occident, via les médias, n'a jamais été aussi grande. D'autre part, la dénonciation de l'hypocrisie nous renseigne aussi sur le mode de gestion des comportements qui peuvent émerger actuellement au Maghreb - sans prétendre que la consommation d'alcool en fasse partie. Admettre que l'on puisse boire, fumer ou fréquenter des personnes du sexe opposé à condition de n'en rien dire, pousse certains individus à se construire sous des identités multiples et disjointes entre elles : un visage pour la famille, un autre au travail ou en public, et un "véritable» visage dans les relations amicales entre égaux. Il n'y a là rien de bien spécifique au Maghreb, mais l'on peut se demander jusqu'à quel point cette situation est tenable sans atteindre la place publique un jour ou l'autre.

\section{BIBLIOGRAPHIE}

ABU NAWWAS, 1979, Le vin, le vent, la vie, anthologie de poèmes traduits et présentés par Vincent Monteil, Paris, Sindbad.

BENKHEIRA M. H., 1985, « Ivrognerie, religiosité et sport dans une ville algérienne (Oran) », Archives de sciences sociales des religions, 59/1, p. 131-151. 
BOZON M., HÉRAN F., 1987-1988, « La découverte du conjoint », Population.

BUISSON-FENET E., 1996, « Des musiques et des Saints, propos de Reda Doumaz, chanteur cha'abi », Maghreb-Machrek, n 154.

CARLIER O., 1990, «Le café maure. Sociabilité masculine et effervescence citoyenne », Annales ESC, $\mathrm{n}^{\circ} 4$, p. 975-1003.

CHEBBI L, 1985, La fêlure, Tunis, Éditions Salammbô.

FERRIE J.-N., 1991, « Évidence de l'individu », dans ESSID Y, L'individu au:Maghreb, Tunis, Beit el Hikma, Ed. T.S.

GOFFMAN E., 1973, La présentation de soi, Paris, Minuit.

HERPIN N., 1973, Les sociologues américains et le siècle, Paris, PUF.

PAPATAXIARCHIS A. P., 1994, «Émotions et stratégies d'autonomie en Grèce égéenne », Terrain, $\mathrm{n}^{\circ} 22$, p. 5-20.

PETONNET C, 1972, Espace, distance et dimension dans une société musulmane. À propos du bidonville de Douar Doum à Rabat, Paris.

ZANNAD T., 1984, Symboliques corporelles et espaces musulmans, Tunis, Cérès Production.

\section{NOTES}

1. La plupart des exemples de lieux de boissons cités ici concernent principalement la ville de Tunis - suite à un travail sociologique sur les mises en scène de la virilité réalisé entre 1994 et 1995 - et, plus ponctuellement, l'Algérie et le Maroc.

*. Ce texte doit beaucoup au travail de correction d'Hélène et de Charif Kiwan, à l'attention de Fanny Colonna et au travail de Jean-Noël Ferrié, sans oublier Jamel S., Jamel M., Hichem, Mejdi... mes compagnons et amis, qui m'ont fait découvrir Tunis.

2. Voir par exemple l'œuvre d'Abu Nawwas (1979); et plus près de nous, les œuvres des poètes algériens, de Si Mohand à Kateb Yacine.

3. Sur l'histoire des débits de boissons au Maghreb, voir l'article d'Omar Carlier (1990), mais aussi l'article plus général de M. H. Benkheira (1985).

4. Sur la conception de l'identité, voir l'introduction de Jean-Noël Ferrié à ce numéro.

5. Idem.

6. Dans le cinéma tunisien, les scènes de bar restent assez rares, et elles sont quasi inexistantes dans les productions télévisuelles.

7. Voir les caractéristiques des références à l'immoralité de l'Occident selon Jean-Noël Ferrié, dans l'introduction à ce numéro.

8. Citons par exemple le « Garibaldi », le « Florent », le « Rossini », ou encore le « Boléro ».

9. La législation tunisienne concernant la vente de l'alcool a évolué dans un sens plutôt restrictif, phénomène bien connu des autres pays maghrébins. Les horaires d'ouverture des bars sont stricts : de $15 \mathrm{~h}$ à $22 \mathrm{~h}$ d'avril à septembre, de $13 \mathrm{~h}$ à $20 \mathrm{~h}$ ensuite. Pendant le mois de Ramadan ainsi que le vendredi (jour de prière), seuls certains restaurants touristiques restent ouverts.

10. Par exemple, la beuverie dans Omar Gatlato Rejla, de M. Allouache, ou bien dans L'homme de Cendres, de N. Bouzid.

11. Une longue série de travaux ont contribué à mettre en place ce modèle, jusqu'à en faire la principale conception de la masculinité au Maghreb. Citons Bishr Farès (1932) et David Gilmore (1987). 
12. La notion de futuwwa, vertu associée à l'origine à l'homme jeune (fera), désignant par la suite un mouvement corporatif dans une grande partie du monde arabe, a la même consonance : elle caractérise à la fois un esprit chevaleresque et ouvert aux autres, généreux et désintéressé, au service de ses compagnons. Voir le Traité de Futuwwa de Al Sulami, qui en offre plus de deux cents définitions... Par opposition, la muruwwa est la vertu de l'homme mûr, qui évoque la maîtrise de soi, la parole pondérée et l'action soigneusement calculée ; c'est la vertu de l'homme public, dont la parole est écoutée dans les assemblées. L'opposition évoquée ci-dessus entre deux formes de virilité reprend donc les mêmes traits - sans qu'ils soient aussi marqués.

13. Pour une comparaison très éclairante avec le monde grec contemporain, voir l'article de A. P. Papataxiarchis (1994).

14. Je ne veux pas nier qu'il existe aussi une certaine "fascination de l'Occident", chez les jeunes en particulier, mais elle n'a pas de moyen public d'expression quand elle concerne des produits comme l'alcool.

15. Ces sanctions peuvent revêtir un caractère très concret: j'ai vu un jeune homme se faire tancer dans la rue par des passants car il avait négligé, à la sortie du marchand de vin, de cacher ses bouteilles. L'habitude veut que l'on occulte les bouteilles au fond d'un "couffin", soigneusement recouvertes de papier journal.

16. Voir l'entrée 3306 du Dictionnaire arabe-français de Daniel Reig, Larousse.

17. Pour un exemple de comparaison entre ces deux répertoires, voir Emmanuel Buisson-Fenet (1996).

\section{INDEX}

Mots-clés : alcool, identité, Occident, Tunisie 